\title{
Gear Fault Detection Analysis Method Based on Fractional Wavelet Transform and Back Propagation Neural Network
}

\author{
Yanqiang Sun ${ }^{1}$, Hongfang Chen ${ }^{1, ~ *}$, Liang Tang ${ }^{1}$ and Shuang Zhang ${ }^{1}$
}

\begin{abstract}
A gear fault detection analysis method based on Fractional Wavelet Transform (FRWT) and Back Propagation Neural Network (BPNN) is proposed. Taking the changing order as the variable, the optimal order of gear vibration signals is determined by discrete fractional Fourier transform. Under the optimal order, the fractional wavelet transform is applied to eliminate noise from gear vibration signals. In this way, useful components of vibration signals can be successfully separated from background noise. Then, a set of feature vectors obtained by calculating the characteristic parameters for the de-noised signals are used to characterize the gear vibration features. Finally, the feature vectors are divided into two groups, including training samples and testing samples, which are input into the BPNN for learning and classification. Experimental results showed that this gear fault detection analysis method could well maintain the useful signal components related to gear faults and effectively extract the weak fault feature. The accuracy rate reached $96.67 \%$ in the identification of the type of gear fault.
\end{abstract}

Keywords: Gear fault detection preparation, factional wavelet transform, back propagation neural network.

\section{Introduction}

With the rapid development of modern industry, mechanical equipment is becoming more complex and efficient. A gear is a key component for motion and power transmission and a strict gear fault detection procedure is required for ensuring superior transmission performance and quality [Ni, Peng, Stöbener et al. (2019); Goch, Ni, Peng et al. (2017); Chen, Sun, Shi et al. (2016); Amaranth and Praveen Krishna (2012); Shi, Lin and Lin (2013); Samanta (2004); Chen, Sun, Shi et al. (2017); Wiemann, Stein and Kniel (2017)].

For meshing gears, gear faults are always found in the vibration signal. In the production environment, vibration signals not only contain the useful information (gear transmission and the fault information), but also have a large number of strong background noise signals. Therefore, fault feature extraction and pattern recognition are two critical problems to be solved [Sahoo, Laskar, Das et al. (2019); Dadon, Koren, Klein et al. (2019); Park, Kim and $\mathrm{Na}(2019)]$.

\footnotetext{
${ }^{1}$ Beijing Engineering Research Center of Precision Measurement Technology and Instruments, Beijing University of Technology, Beijing, 100124, China.

*Corresponding Author: Hongfang Chen. Email: chf0302@126.com.
} 
The traditional spectrum analysis method based on Fast Fourier Transform (FFT) is only suitable for the analysis of stationary signals. However, measured dynamic signals often contain a large number of non-stationary signal components. The time-frequency analysis method, a new method developed in the fault analysis field, can overcome the disadvantage of the traditional spectrum analysis method. Therefore, many time-frequency analysis methods are proposed, including Wavelet Transform (WT), Fractional Fourier Transform (FRFT), Short-time Fractional Fourier Transform (SFRFT), Radon-Wigner Transform and Fractional Wavelet Transform (FRWT). However, WT is only limited in the timefrequency domain [Bajric, Zuber, Krimpas et al. (2016); Shao and Deng (2018); Mallat (1989); Peng and Chu (2004); Gargour, Gabrea and Ramachandran (2009)]. FRFT cannot characterize local features of signals [Almeida (1994); Yuan (2008); Narayanan and Prabhu (2003)]. The high resolution of SFRFT cannot be achieved simultaneously in the time domain and fractional domain [Tao, Li and Wang (2010)]. Radon-Wigner transform is based on quadratic transformations, which have cross terms [Wood and Barry (1994)].

In addition, method of selection of amplitudes of frequency (MSAF), method of selection of amplitudes of frequency-multiexpanded (MSAF-MULTIEXPANDED), shortened method of frequencies selection (SMOFS) and shortened method of frequencies selectionMultiexpanded (SMOFS-MULTIEXPANDED) are feature extraction methods [Adam (2016, 2018, 2019); Adam and Witold (2018)]. There is no need to connect a measuring sensor with the machine for acoustic-based measurements, which can analyze acoustic signals in places with limited or no access. However, there are still some problems and difficulties. One of the difficulties to solve was selection of training samples. It can be noticed that the recognition results depended on selected training samples. All samples are measured by one microphone. If the acoustic signal is measured by another type of microphone, then it can cause errors of recognition. The second of the difficulties to solve was the testing (classification) of a new unknown test samples. The third of the difficulties to solve was background noises. They cannot work for a machine that does not generate acoustic signals. Background noises can be also problem, if we analyze several devices in one place and at the same time. What's more, the acoustic-based analysis was expensive. Cons of this solution are the higher cost and size of the computer.

Because of the complex structure and various defect forms of gear transmission system, the pattern recognition based on artificial intelligence has become an inevitable trend.

Fractional Wavelet Transform (FRWT), proposed originally by Mendlovic in 1997, combines wavelet transform with fractional order theory and have significant advantages in signal processing [Mendlovic, Zalevsky and Mas (1997); Chen and Zhao (2005); Zhuo (2018)]. Based on the wavelet transform and the fractional Fourier transform, the Fractional wavelet transform (FRWT) is applied to the decomposition and reconstruction of the signals. And it is proved that the transform satisfies the law of conservation of energy. The fractional wavelet transform domain is related to the time-frequency domain to some extent. So essentially, the synthesis and decomposition of fractal-order wavelet transform is a consistent transformation in the time-frequency domain. Compared with the traditional time-frequency analysis method, the fractional wavelet transform has an important advantage that it has a single adjustable variable, namely the fractional-order. Therefore, the fractional wavelet transform can be better used to filter various random signals, which 
obtain good analysis and processing results.

Back Propagation neural network (BPNN) proposed by Rumelhart and McCelland in 1986 [Qin, Yang and Cheng (2008)] is a multi-layer feed-forward neural network trained by error back-propagation algorithm, which has the advantages of fast computation and low memory consumption [Samanta (2004); Samanta, Al-Balushi and Al-Araimi (2003)]. BPNN can learn and store input-output mapping relationships. It is not required to reveal the mathematical equations describing the mapping relations. Therefore, it avoids the deficiency of traditional fault analysis method based on sideband. Back Propagation neural network has good self-learning and classification ability, which can be used to identify fault types in fault diagnosis [Zhao (2018); Yang, Duan, Yu et al. (2018); Huang, Liang, Lei et al. (2019); Wang, Ji and Ji (2019)].

In this paper, a gear fault detection analysis method based on Fractional Wavelet Transform and Back Propagation Neural Network is proposed. The optimal order on two-dimensional plane is obtained from mixed vibration signals though discrete fractional Fourier transform. Then, the vibration signal is subjected to the fractional wavelet transform with the optimal order. The fractional wavelet reconstruction is completed by combining low frequency coefficient with high frequency coefficient after threshold quantization. In the de-noised signal, energy values in the frequency band are extracted to build feature vectors, which can be used in BPNN to further realize the intelligent identification of the faulty gear.

With FRWT and BPNN, the method can quickly achieve the gear fault detection. Furthermore, the gear quality can be checked according to the detection results. Therefore, this method may be used as a powerful analysis method for the quality diagnosis of gears.

\section{Fundamental principles}

\subsection{Fractional wavelet transform}

FRFT has attracted wide attention from many researchers in various fields, especially in signal processing. The discrete form of fractional Fourier transform is required in processing digital signals in engineering. The discrete order fractional Fourier transform of signal is defined as:

$X_{p}\left(\frac{m}{2 \Delta x}\right)=A_{\alpha} e^{j \frac{1}{2}\left(\frac{m}{2 \Delta x}\right)^{2}(\cot \alpha-\csc \alpha)} \sum_{n=-N}^{N}\left[x\left(\frac{n}{2 \Delta x}\right) e^{j \frac{1}{2}\left(\frac{m}{2 \Delta x}\right)^{2}(\cot \alpha-\csc \alpha)}\right] e^{j \frac{1}{2}\left(\frac{m-n}{2 \Delta x}\right)^{2} \csc \alpha}$

where $A_{\alpha}=\sqrt{1-j \cot \alpha / 2 \pi}$; $\alpha$ is a rotation angle and $\alpha=p \pi / 2, p$ is the order of discrete order fractional Fourier transform, which is an arbitrary real number; $\Delta x$ is the signal bandwidth; $N$ indicates sample length, $N=(\Delta x)^{2}$.

FRWT aims to improve the multi-resolution wavelet analysis theory [Huang (1988)]. For the signal $x(t)$, its fractional wavelet transform is expressed as:

$W^{(p)}(a, b)=\int_{-\infty}^{+\infty} \int_{-\infty}^{+\infty} B_{p}\left(t, t^{\prime}\right) x\left(t^{\prime}\right) \psi_{a b}^{*}(t) d t^{\prime} d t$

where $\psi_{a b}^{*}(t)$ is a wavelet basis function; $a$ is the dilation factor; $b$ is the translation factor; $B_{p}\left(t, t^{\prime}\right)$ is a kernel function: 


$$
B_{p}\left(t, t^{\prime}\right)=\sqrt{2} \exp \left[-\pi\left(t^{2}+t^{\prime 2}\right)\right] \times \sum_{n=0}^{\infty} \frac{i^{-p n}}{2^{n} n !} H_{n}(\sqrt{2 \pi} t) H_{n}\left(\sqrt{2 \pi} t^{\prime}\right)
$$

where $H_{n}$ is $n$-order Hermite polynomials; $p$ is the order of FRWT and ranges from 0 to 1 . The reconstruction formula of fractional wavelet transform is:

$$
X(t)=\frac{1}{C} \int_{-\infty}^{+\infty} \int_{-\infty}^{+\infty} \int_{-\infty}^{+\infty} \frac{1}{a^{3}} W^{(p)}(a, b) B_{-p}\left(t, t^{\prime}\right) \times \psi_{a b}^{*}(t) d a d b d t^{\prime}
$$

\subsection{Back propagation neural network}

Back Propagation neural network, one of the mature neural networks, is a multi-layer network trained with the weights of nonlinear differentiable functions [Li, Han and $\mathrm{Yu}$ (2017); Jiang, Zhang, Xuan et al. (2016); Xu, Zhang, Wang et al. (2015)]. The learning rule of BPNN is a gradient descent algorithm. The weights and thresholds of the network are adjusted constantly by back-propagation to minimize the square error of the network. As shown in Fig. 1, the BPNN is composed of an input layer, a hidden layer and an output layer. The number of input layer nodes is determined by the number of characteristic values contained in a feature vector. The number of output layer nodes is determined by gear fault types. For the determination of the number of hidden layer nodes, there is no definite theoretical rule. In the specific design, a practical approach is to train and compare different neurons, so it needs to properly add a little margin. Moreover, the number of hidden layer nodes is affected by the number of input layer nodes, the number of output layer nodes, the target type number and other factors. In this paper, the number of hidden layer nodes is determined as:

$n=\frac{n_{I}+\left(n_{O}, n_{C}\right)_{\max }}{2}$

where $n$ is the number of hidden layer nodes; $n_{I}$ is the number of input layer nodes; $n_{O}$ is the number of output layer nodes; $n_{C}$ is the number of target classification.

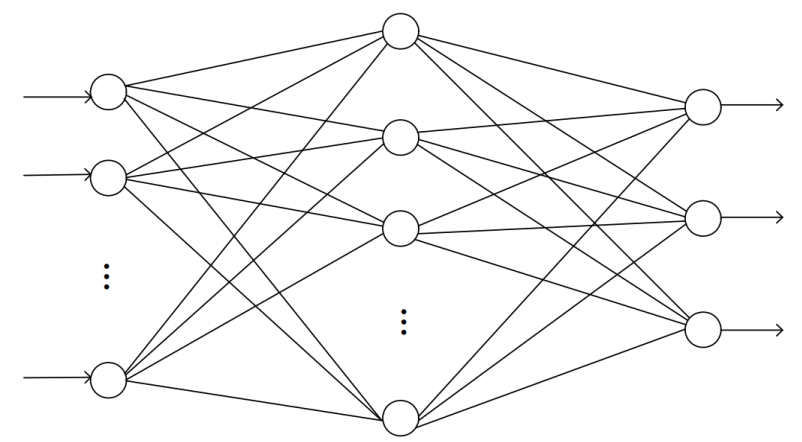

Input Layer Hidden Layer Output Layer

Figure 1: Back Propagation neural network structure 


\section{Intelligent analysis method of gear fault detection}

\subsection{Noise removal based on fractional wavelet transform}

The fractional wavelet transform combines the advantages of fractional Fourier transform and wavelet transform. So it highlights many advantages including multi-resolution analysis in wavelet transform, time-frequency focusing of fractional Fourier transform and so on. Though de-noising the gear vibration signal containing background noise, the deficiency of single transformation is overcome to ensure the consistency and validity of time-frequency resolution. Therefore, it is suitable to extract and analyze gear fault features. The flow chart of the fractional wavelet transform is shown in Fig. 2.

The mixed vibration signal $s(t)$ is collected by a sensor, $s(t)=x(t)+w(t)$. The signal $s(t)$ contains the useful component of the gear vibration signal $x(t)$ and the background noise signal $w(t)$.The specific implement method is described as follows:

1. The mixed vibration signal $s(t)$ is subjected to the discrete fractional Fourier transform with the order $p$. Signal energy is distributed in two-dimensional $p-u$ plane.

2. The peak point is searched on two-dimensional plane $(p, u)$ formed by in Step 1.The optimal order $p_{\text {opt }}$ is obtained by calculating $\underset{p, u}{\arg \max }\left|X_{p}(u)\right|^{2}$.

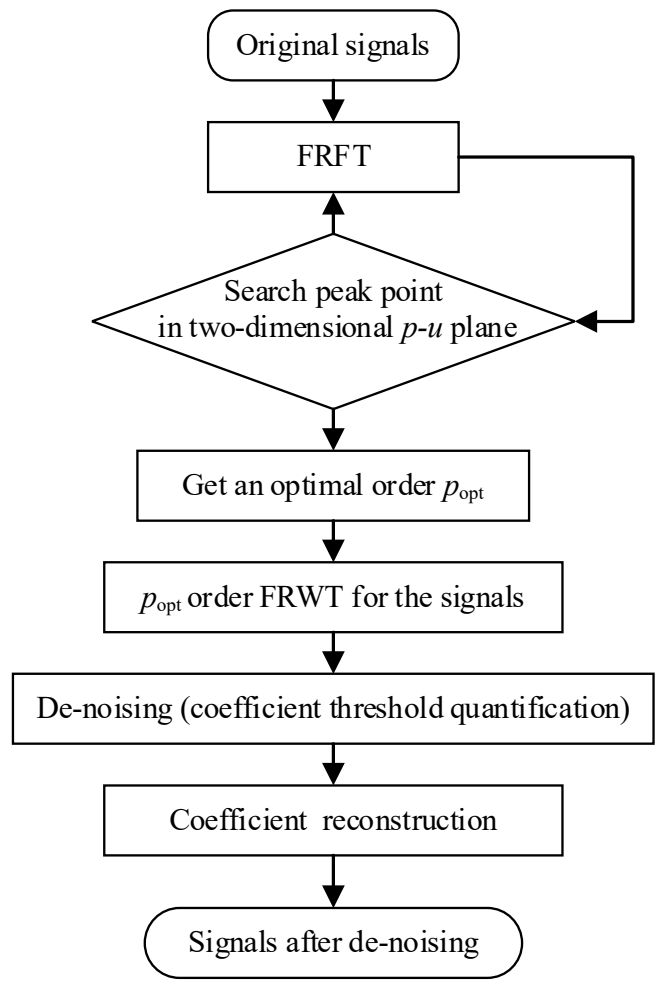

Figure 2: Flow chart of the fractional wavelet transform 
3. The appropriate wavelet decomposition order is selected as $N$. The mixed vibration signal $s(t)$ is subjected to the fractional wavelet transform with the order $p_{o p t}$. Its $N^{\text {th }}$ low frequency coefficient $c a_{N}$ and high frequency coefficients from $1^{\text {st }}$ to $N^{\text {th }}$ layer $c d_{1}, c d_{2}, \cdots c d_{N}$ are obtained.

4. The high frequency coefficients $\left(c d_{1}, c d_{2}, \cdots c d_{N}\right)$ is subjected to threshold processing. The threshold is selected by $\eta=\operatorname{sqrt}(2 * \log (n *(n) / \log (2)))$, where $n$ is the number of wavelet coefficients. The threshold function is

$$
\tilde{\omega}_{i, j}=\left\{\begin{array}{cc}
\operatorname{sgn}\left(\omega_{i, j}\right)\left(\left|\omega_{i, j}\right|-\eta\right)\left|\omega_{i, j}\right| & \geq \eta \\
0 & \left|\omega_{i, j}\right|<\eta,
\end{array}\right.
$$

where $\eta$ is the threshold coefficient and $\omega_{i, j}$ is the wavelet decomposition coefficient. After threshold processing, the high frequency coefficients are respectively denoted as $c d_{1}^{\prime}, c d_{2}^{\prime}, \cdots c d_{N}^{\prime}$.

5. Through combining $N^{\text {th }}$ low frequency coefficient $c a_{N}$ with the high frequency coefficient $\left(c d_{1}^{\prime}, c d_{2}^{\prime}, \cdots c d_{N}^{\prime}\right)$ after threshold quantization, $p_{\text {opt }}$-order fractional wavelet reconstruction is completed. Therefore, it can get the useful gear transmission signal component and realize the separation from background noise.

\subsection{Signal feature}

The 10 time-domain statistical parameters are selected as the characteristic parameters of gear vibration signal after de-noising, including 5 dimensional parameters and 5 dimensionless parameters. The formulas are shown in Tab. 1.

Table 1: Time-domain statistical parameters of signal and its calculation formulas

\begin{tabular}{ccc}
\hline No. & $\begin{array}{c}\text { Dimensional } \\
\text { parameters }\end{array}$ & Calculation formulas \\
\hline 1 & Peak value & $X_{p p v}=X_{\max }-X_{\min }$ \\
2 & Mean value & $u_{x}=\frac{1}{N} \sum_{n=1}^{N-1} x(n)$ \\
3 & $\begin{array}{c}\text { Root mean } \\
\text { square value }\end{array}$ & $X_{r m s}=\sqrt{\frac{1}{N} \sum_{n=1}^{N-1} x^{2}(n)}$ \\
4 & Skewness & $\alpha=\frac{\bar{x}-M_{0}}{\sigma}$ \\
5 & Kurtosis & $\beta=\frac{1}{N} \sum_{i=1}^{N}\left(\frac{x_{i}-\bar{x}}{\sigma_{i}}\right)^{4}$ \\
6 & Waveform index & $K=\frac{X_{r m s}}{\left|u_{s}\right|}$ \\
\hline
\end{tabular}




\begin{tabular}{lll}
\hline 7 & Peak index & $C=\frac{X_{\max }}{X_{r m s}}$ \\
8 & Pulse index & $I=\frac{X_{\max }}{\left|u_{s}\right|}$ \\
9 & Margin index & $=\frac{X_{\max }}{X_{r}}$ \\
10 & Kurtosis index & $K_{v}=\frac{\beta}{X_{r m s}^{4}}$
\end{tabular}

Notes: $X_{\max }$ is the maximum of the signal; $X_{\min }$ is the minimum of the signal; $x(n)$ is the signal at the sampling point $n ; N$ is total data length; $\bar{x}$ is average value; $M_{0}:$ is median or mode; $\sigma$ is standard deviation; $\left|u_{s}\right|$ is average amplitude.

\subsection{Gear fault pattern recognition}

The feature vectors in Section 3.2 are used as the sample inputs in BPNN for training and recognition. In this paper, the neural network contains a hidden layer. After training a large number of feature vectors, the weight matrix and threshold matrix are recorded. The training process is completed in order to recognize intelligently gear faults. The flow chart is shown in Fig. 3.

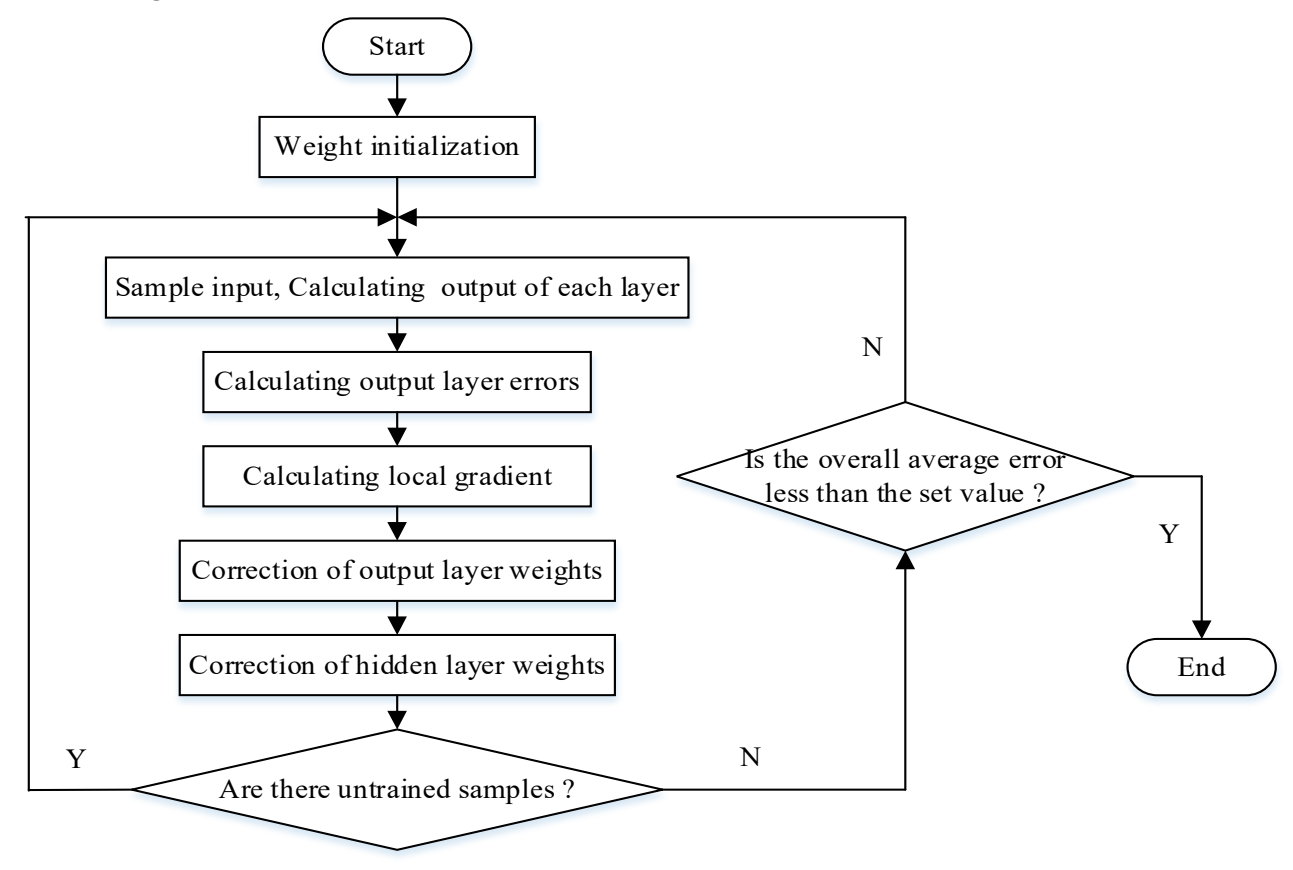

Figure 3: Flow chart of BPNN

Sample training steps of BPNN are described as follows: 
1. Initialize weight matrix and threshold. The mode counter and training counter of the sample are set as 1 . The error, learning rate, and the minimum precision and the maximum number of iterations allowed in the network are initialized.

2. Input training samples and calculate the output of the hidden layer $o_{k}$ and the output of the output layer $y_{i}$ :

$$
\begin{aligned}
& y_{i}=\phi\left(\text { net }_{i}\right)=\phi\left(\sum_{j=1}^{M} v_{i j} x_{j}+\theta_{i}\right) \\
& o_{k}=\psi\left(\text { net }_{k}\right)=\psi\left(\sum_{i=1}^{Q} w_{k i} y_{i}+a_{k}\right)=\psi\left(\sum_{i=1}^{Q} w_{k i} \phi\left(\sum_{j=1}^{M} v_{i j} x_{j}+\theta_{i}\right)+a_{k}\right)
\end{aligned}
$$

3. Calculate the sample output error $E_{p}$.

$E_{p}=\frac{1}{2} \sum_{k=1}^{L}\left(t_{k}-o_{k}\right)^{2}$

The network has different errors for different samples. The total error of the system $E$ can be obtained by accumulating output errors of all samples:

$E=\frac{1}{2} \sum_{p=1}^{P} \sum_{k=1}^{L}\left(t_{k}^{p}-o_{k}^{p}\right)^{2}$

4. Check whether the training process of all samples are completed. If not, go to Step 2.

Otherwise, go to Step 5.

5. Adjust the weights and thresholds of each layer according to the following formulas:

$$
\begin{aligned}
& \Delta w_{k i}=\eta \sum_{p=1}^{P} \sum_{k=1}^{L}\left(t_{k}^{p}-o_{k}^{p}\right) \cdot \psi^{\prime}\left(\text { net }_{k}\right) \cdot y_{i}, \\
& \Delta a_{k}=\eta \sum_{p=1}^{P} \sum_{k=1}^{L}\left(t_{k}^{p}-o_{k}^{p}\right) \cdot \psi^{\prime}\left(\text { net }_{k}\right), \\
& \Delta v_{i j}=\eta \sum_{p=1}^{P} \sum_{k=1}^{L}\left(t_{k}^{p}-o_{k}^{p}\right) \cdot w_{k i} \cdot \phi^{\prime}\left(\text { net }_{i}\right) \cdot x_{j}, \\
& \Delta \theta_{i}=\eta \sum_{p=1}^{P} \sum_{k=1}^{L}\left(t_{k}^{p}-o_{k}^{p}\right) \cdot w_{k i} \cdot \phi^{\prime}\left(\text { net }_{i}\right),
\end{aligned}
$$

The iterative increment of weights and the thresholds for each neuron updates the weight and threshold for the next round of network learning and training. Its update formula is

$$
w_{k i}(n+1)=w_{k i}(n)+\Delta w_{k i}, \quad v_{i j}(n+1)=v_{i j}(n)+\Delta v_{i j},
$$


6. Check whether the total error of the network meets the accuracy requirements. If it is less than the minimum precision allowed by the network, the training is completed. Otherwise, it will return to Step 2 and continue.

7. Check whether the number of iterations reach its maximum. If it is less than the maximum of iterations, the training returns to Step 2 and continues. Otherwise, the training is completed.

\section{Experiments}

In this paper, gear vibration signals were collected by the test rig (Fig. 4). It is composed of a gear rolling inspection machine, acceleration sensors and testing gears (Fig. 5). The gears are a pair of spiral bevel gears, whose driving and driven wheel share a gear tooth number of 17 , with a transmission ratio of 1:1. The rotation speed of driving wheel is 750 $1000 \mathrm{rpm}$. The sampling frequency of the vibration signal sound is $20 \mathrm{kHz}$. Parameters of driving and driven wheel are shown in Tab. 2.

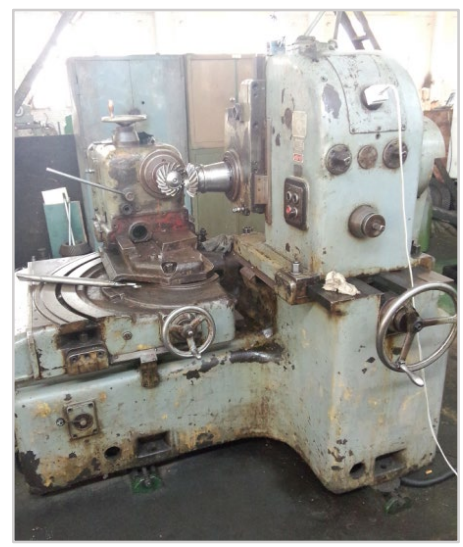

Figure 4: Test rig diagram

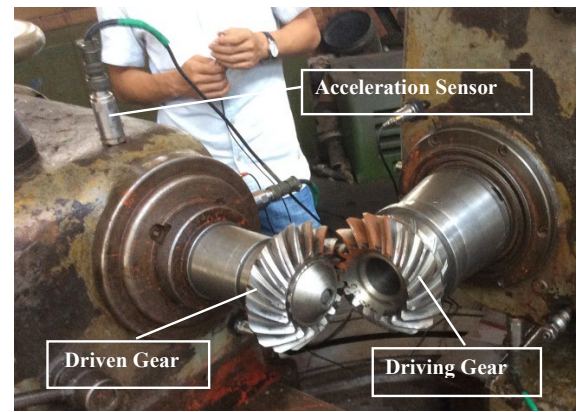

Figure 5: Test rig and acceleration sensors

Table 2: Parameters of driving gear and driven gear

\begin{tabular}{|c|c|c|c|c|c|}
\hline Parameters & Module & Whole depth & Number of teeth & Pressure angle & $\begin{array}{c}\text { Helical } \\
\text { angle }\end{array}$ \\
\hline $\begin{array}{l}\text { Driving } \\
\text { gear }\end{array}$ & 8.034483 & $15.17 \mathrm{~mm}$ & 17 & $20^{\circ}$ & $35^{\circ}$ \\
\hline $\begin{array}{c}\text { Driven } \\
\text { gear }\end{array}$ & 8.034483 & $15.17 \mathrm{~mm}$ & 17 & $20^{\circ}$ & $35^{\circ}$ \\
\hline Parameters & Spiral direction & $\begin{array}{l}\text { Addendum } \\
\text { coefficient }\end{array}$ & \multicolumn{2}{|l|}{$\begin{array}{l}\text { Addendum Clearance } \\
\text { coefficient }\end{array}$} & $\begin{array}{c}\text { Tangential } \\
\text { modification } \\
\text { coefficient }\end{array}$ \\
\hline $\begin{array}{l}\text { Driving } \\
\text { gear }\end{array}$ & levorotation & 0.8 & 0.188 & \multicolumn{2}{|c|}{-0.064} \\
\hline $\begin{array}{l}\text { Driven } \\
\text { gear }\end{array}$ & dextrorotation & 0.8 & 0.188 & \multicolumn{2}{|c|}{+0.064} \\
\hline
\end{tabular}


There are two acceleration sensors in the experimental device, and they are mounted on the driving gear base and the driven gear base respectively. The tested gears include normal gears, gears with reduced meshing clearance (reduced by $0.035 \mathrm{~mm}$ ), and gears with increased meshing clearance (increased by $0.035 \mathrm{~mm}$ ).

As shown in Fig. 6, it can be seen that there are obvious modulation phenomena in the time and frequency domain of the gear vibration signals obtained from the experimental measurements. Ambient noise obscures the vibration signal produced by the meshing of gears, so it is not effective to distinguish the three gear types according to the time and frequency domain curve.
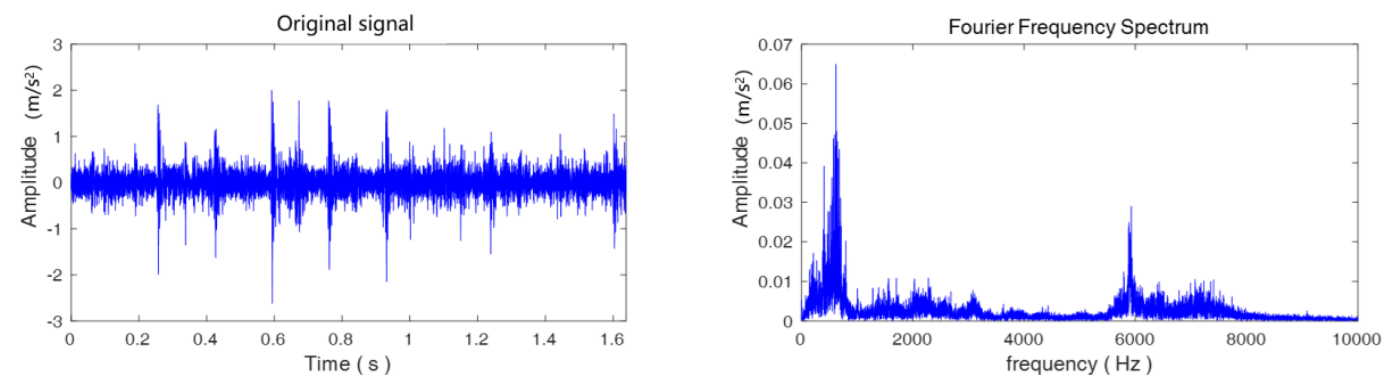

(a)
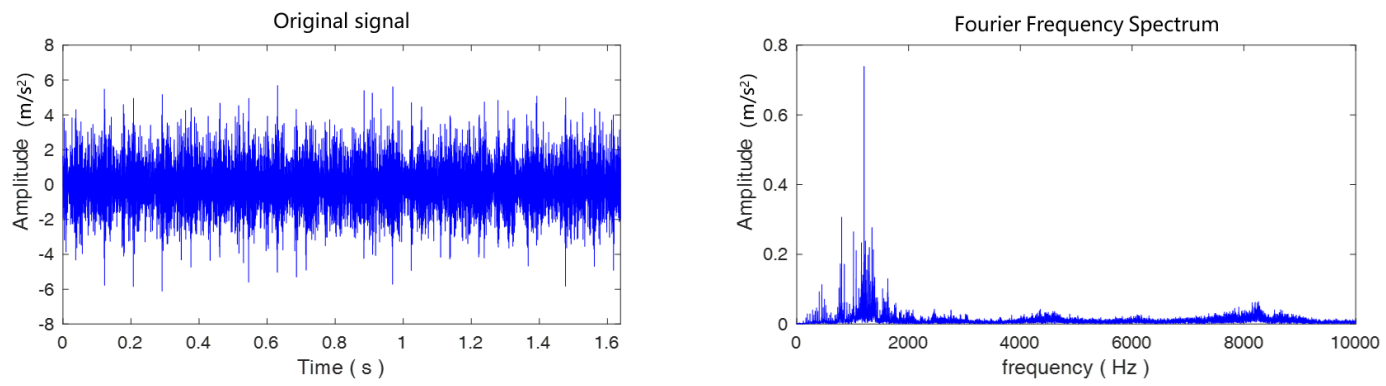

(b)
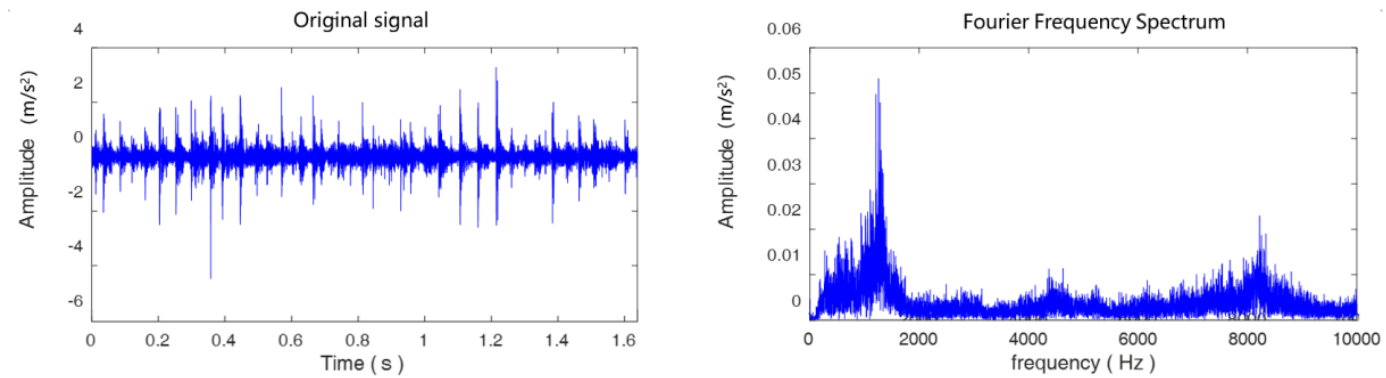

(c)

Figure 6: Gear vibration signals. (a) Original signals and Fourier frequency spectrum of the normal gears; (b) Original signals and Fourier frequency spectrum of the gears with reduced meshing clearance; (c) Original signals and Fourier frequency spectrum of the gears with increased meshing clearance 
The vibration signals of the above three kinds of gears are analyzed by the method proposed in this paper. The flow chart is shown in Fig. 7.

Step 1: For the collected gear vibration signal, discrete fractional Fourier transform with order $p$ is applied to distribute signal energy in two-dimensional $p-u$ plane. The order $p$ increases from 0 to 2 with a step size of 0.01 . Through the peak value search and calculation, an optimal order $p_{\text {opt }}$ is determined on the 2-dimensional plane. In the experiment, an optimal order $p_{\text {opt }}$ for the gear vibration signal with gears with reduced meshing clearance is 1.103 .

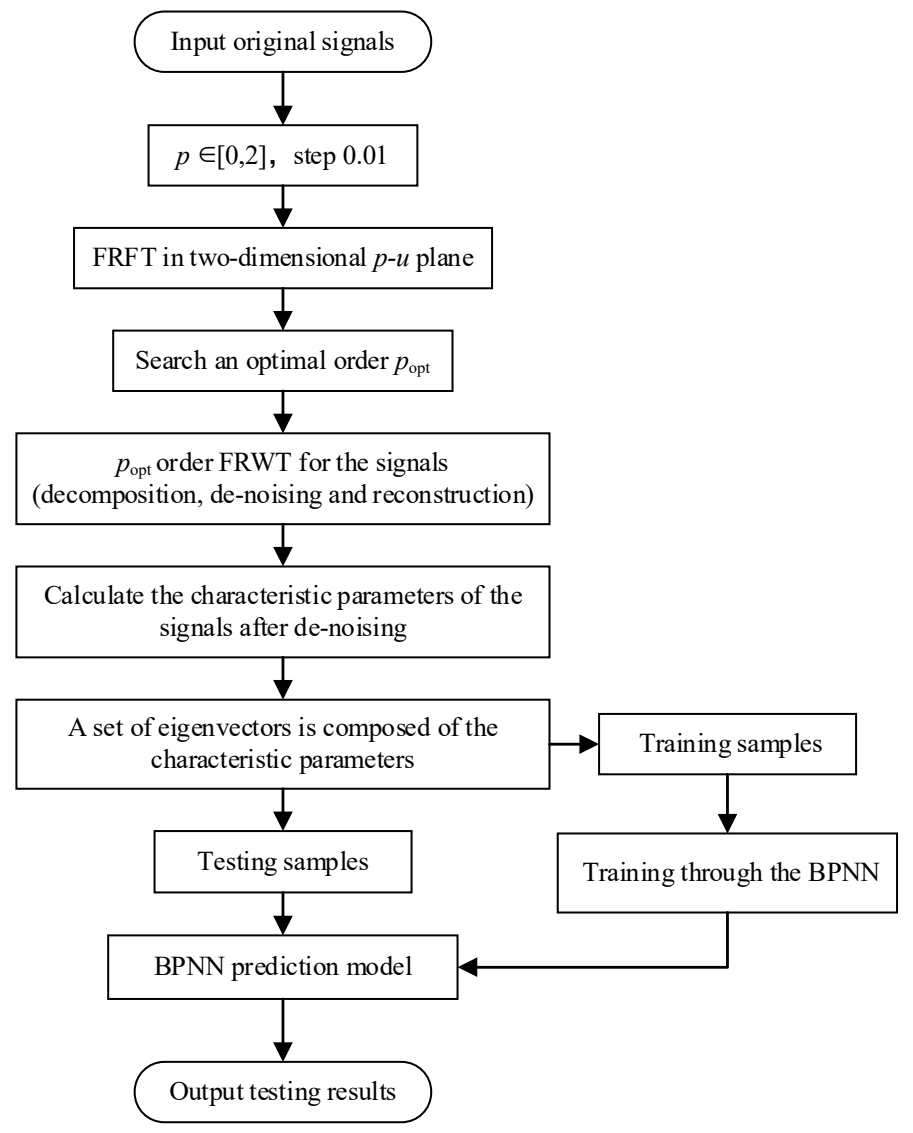

Figure 7: Diagram of the methodology based on FRWT and BPNN

Step 2: Fractional wavelet transform is applied in the signal filtering to get useful gear vibration signal, which is decomposed and reconstructed by db6 3-layer wavelet packet to obtain the decomposition energy spectrum. Frequency of gear vibration signal ranges from 0 to $2000 \mathrm{~Hz}$ and the sampling frequency $f_{s}$ is $20 \mathrm{kHz}$. Therefore, when $k$-layer wavelet packet decomposition is performed with the gear vibration signal, the node bandwidth at the $k^{\text {th }}$ layer is 
$f_{B W}=\frac{f_{s}}{2^{k+1}}$,

where the frequency range of each node is:

$m f_{B W} \sim(m+1) f_{B W}, m=0,1,2, \ldots, 2^{k}-1$.

where $m$ represents the node number at the $k^{\text {th }}$ layer in wavelet decomposition.

And the de-noised gear signals are shown in Fig. 8.
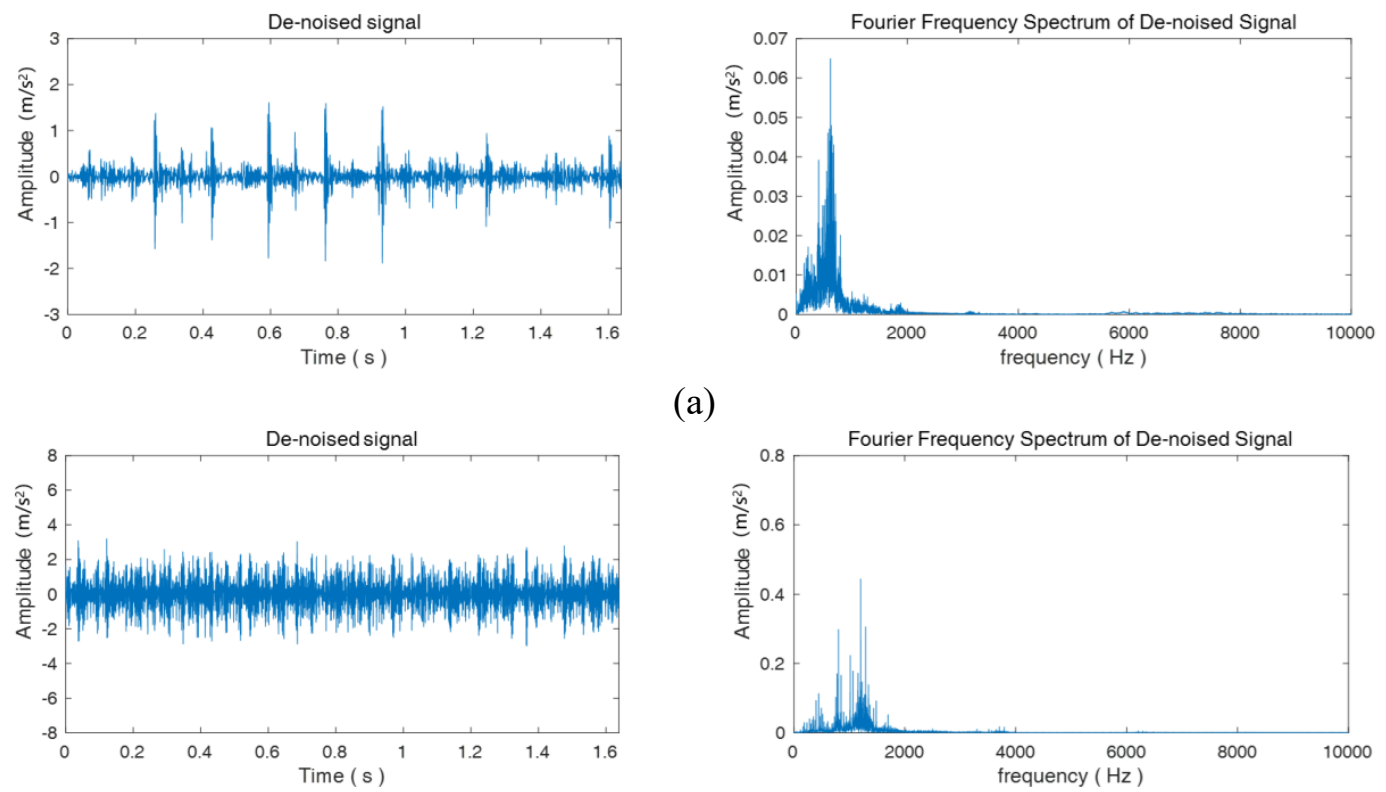

(a)

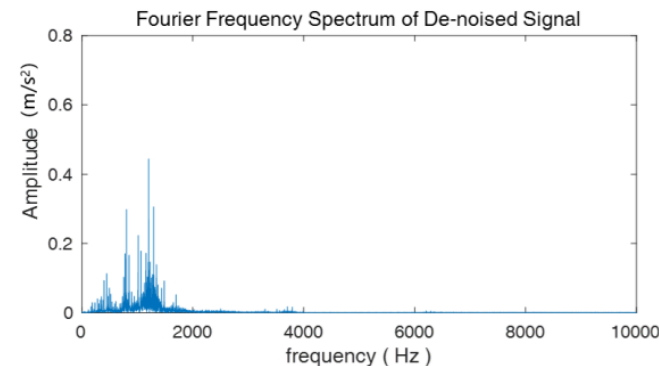

(b)
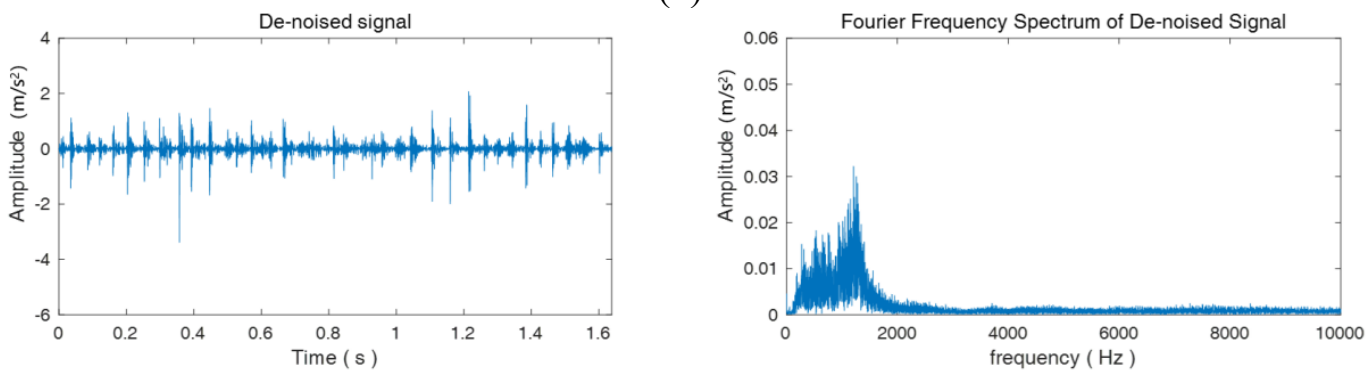

(c)

Figure 8: De-noised gear signals. (a) De-noised signals and Fourier frequency spectrum of the normal gears; (b) De-noised signals and Fourier frequency spectrum of the gears with reduced meshing clearance; (c) De-noised signals and Fourier frequency spectrum of the gears with increased meshing clearance 
Step 3: The statistical parameters are selected as the characteristic parameters of the vibration signal after de-noising, including peak value, mean value, root mean square value, skewness, kurtosis, waveform index, peak index, pulse index, margin index and kurtosis index (Fig. 9).

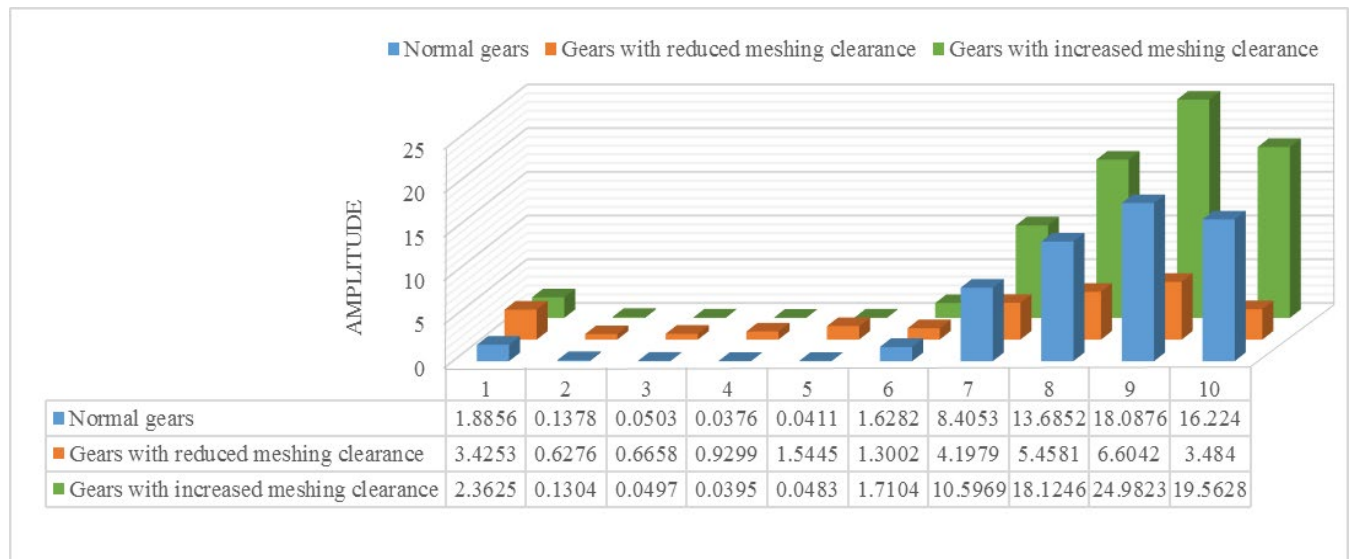

Note: Units of the parameters 1 through 5 are respectively $\mathrm{m} / \mathrm{s}^{2}, \mathrm{~m} / \mathrm{s}^{2}, \mathrm{~m} / \mathrm{s}^{2},\left(\mathrm{~m} / \mathrm{s}^{2}\right)^{3},\left(\mathrm{~m} / \mathrm{s}^{2}\right)^{4}$; the parameters 6 through 10 are dimensionless parameters.

Figure 9: Characteristic parameters of the vibration signal after de-noising

Step 4: The BPNN is used to classify and study 60 groups of the feature vectors of the vibration signals after de-noising. The feature vectors are divided into two types: 30 groups of training samples and 30 groups of testing samples. Both training samples and testing samples contain 10 groups of normal gears, 10 groups of gears with reduced meshing clearance, and 10 groups of gears with increased meshing clearance. For the BPNN, the node numbers of input layer, hidden layer and output layer are respectively 10, 7 and 3. The error condition during training is that the error is less than 0.0001 or the learning times are not less than 10000 times. After training, 30 groups of testing samples are input into the BPNN. The numbers 1-10 indicate the feature vectors of the vibration signals for normal gears. The numbers 11-20 indicate the feature vectors of the vibration signals for gears with reduced meshing clearance (reduced by $0.03 \mathrm{~mm}$ ). The numbers $21-30$ indicate the feature vectors of the vibration signals for gears with increased meshing clearance (increased by $0.03 \mathrm{~mm}$ ). The identification results of the BPNN are shown in Tab. 4 .

As shown in Tab. 4, the training and prediction results of three gear-fault types using the BPNN can reach an accuracy of $96.67 \%$.

Table 4: Identification results of the BPNN

\begin{tabular}{cccccc}
\hline No. & \multicolumn{2}{c}{ BPNN identification values } & Identification results & T / F \\
\hline 1 & 0.9724 & 0.0538 & 0.0107 & Normal gear & $\mathrm{T}$ \\
2 & 0.9392 & 0.0258 & 0.0380 & Normal gear & $\mathrm{T}$ \\
3 & 0.9677 & 0.0301 & 0.0184 & Normal gear & $\mathrm{T}$ \\
4 & 0.1712 & 0.0224 & 0.7921 & Gear with increased meshing clearance & $\mathrm{F}$ \\
5 & 0.9586 & 0.0281 & 0.0246 & Normal gear & $\mathrm{T}$ \\
\hline
\end{tabular}




\begin{tabular}{cccccc}
\hline 6 & 0.9640 & 0.0764 & 0.0107 & Normal gear & $\mathrm{T}$ \\
7 & 0.9214 & 0.0283 & 0.0467 & Normal gear & $\mathrm{T}$ \\
8 & 0.9301 & 0.0153 & 0.0607 & Normal gear & $\mathrm{T}$ \\
9 & 0.9742 & 0.0429 & 0.0117 & Normal gear & $\mathrm{T}$ \\
10 & 0.8898 & 0.0288 & 0.0645 & Normal gear & $\mathrm{T}$ \\
11 & 0.0289 & 0.9746 & 0.0010 & Gear with reduced meshing clearance & $\mathrm{T}$ \\
12 & 0.0285 & 0.9748 & 0.0021 & Gear with reduced meshing clearance & $\mathrm{T}$ \\
13 & 0.0316 & 0.9729 & 0.0015 & Gear with reduced meshing clearance & $\mathrm{T}$ \\
14 & 0.0259 & 0.9772 & 0.0013 & Gear with reduced meshing clearance & $\mathrm{T}$ \\
15 & 0.0277 & 0.9758 & 0.0010 & Gear with reduced meshing clearance & $\mathrm{T}$ \\
16 & 0.0271 & 0.9765 & 0.0010 & Gear with reduced meshing clearance & $\mathrm{T}$ \\
17 & 0.0269 & 0.9767 & 0.0014 & Gear with reduced meshing clearance & $\mathrm{T}$ \\
18 & 0.0305 & 0.9775 & 0.0021 & Gear with reduced meshing clearance & $\mathrm{T}$ \\
19 & 0.0238 & 0.9785 & 0.0011 & Gear with reduced meshing clearance & $\mathrm{T}$ \\
20 & 0.0230 & 0.9786 & 0.0011 & Gear with reduced meshing clearance & $\mathrm{T}$ \\
21 & 0.0478 & 0.0105 & 0.9691 & Gear with increased meshing clearance & $\mathrm{T}$ \\
22 & 0.0423 & 0.0101 & 0.9737 & Gear with increased meshing clearance & $\mathrm{T}$ \\
23 & 0.0420 & 0.0201 & 0.9739 & Gear with increased meshing clearance & $\mathrm{T}$ \\
24 & 0.1796 & 0.0115 & 0.8468 & Gear with increased meshing clearance & $\mathrm{T}$ \\
25 & 0.0420 & 0.0101 & 0.9745 & Gear with increased meshing clearance & $\mathrm{T}$ \\
26 & 0.0411 & 0.0108 & 0.9738 & Gear with increased meshing clearance & $\mathrm{T}$ \\
28 & 0.0006 & 0.0578 & 0.9323 & Gear with increased meshing clearance & $\mathrm{T}$ \\
29 & 0.0420 & 0.0124 & 0.9731 & Gear with increased meshing clearance & $\mathrm{T}$ \\
\hline $\mathrm{N}$ & 0.0013 & 0.0584 & 0.9361 & Gear with increased meshing clearance & $\mathrm{T}$ \\
$\mathrm{T}$ & Gear with increased meshing clearance & $\mathrm{T}$ \\
\hline
\end{tabular}

Note: Ideally, the recognition result of the normal gear is [ 1000 ; the recognition result of the gear with reduced meshing clearance is $\left[\begin{array}{lll}0 & 1 & 0\end{array}\right]$; the recognition result of the gear with increased meshing clearance is $\left[\begin{array}{lll}0 & 0 & 1\end{array}\right]$. Since it is impossible that the simulation results are equal to 0 or 1 , their results need to be adjusted. The results below 0.25 are expressed as 0 and the results above 0.75 are expressed as 1 .

In this paper, a comparative experiment is carried out for the de-noising effect of fractional order transformation. When the feature signals of the above original data are extracted without FRWT for background noise elimination and then recognized by BPNN, the classification accuracy of gear type is only $78 \%$. In addition, support vector machine method is selected to replace BPNN for gear fault pattern recognition. With the same number of samples, the classification accuracy of gear type can only reach $86.67 \%$, as shown in Fig. 10. 


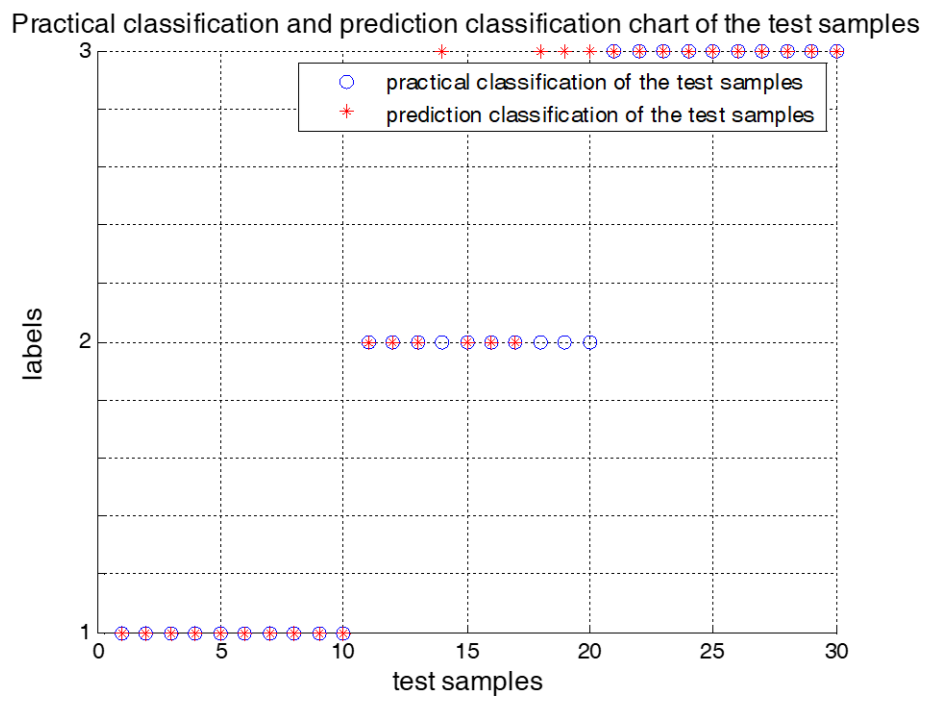

Figure 10: Classification accuracy of gear type based on support vector machine

Experimental results proved that the FRWT proposed in this paper could effectively extract useful signals reflecting the fault feature under a background condition filled with powerful noise. The BPNN can intelligently recognize gear fault types without the artificial manipulation. Combining FRWT and BPNN, the method can quickly achieve the gear fault detection. Furthermore, the gear quality can be checked according to the detection results.

\section{Conclusions}

This paper proposes a gear fault detection analysis method based on FRWT and BPNN. The FRWT method is applied to eliminate noise from the collected gear vibration signal, thus realizing the separation of the useful component of vibration signal from the background noise. A set of feature vectors are obtained by calculating the characteristic parameters for the de-noised signal, which are used in training and classification with the BPNN. The FRWT can extract useful signals to reflect the gear fault features and achieve good elimination effects on background noise. In addition, the automatic recognition of gear fault is realized through the clustering and classification of vibration signal vectors by the BPNN. Therefore, the gear vibration analysis method based on FRWT and BPNN is intelligent, efficient and accurate.

In order to further improve the accuracy and efficiency of gear fault diagnosis, we will continue to do the following work: 1) Increase the number of training samples. It will obtain more prominent gear features, which improve the accuracy of intelligent detection. At the same time, increase the type of gear faults. It will improve the gear fault analysis and intelligent detection of various gear faults. On this basis, we will explore how to further reduce the computational complexity of the algorithm and improve the operation speed, which further improve the accuracy of the algorithm; 2) For practical applications, the key problems to be solved urgently are the location analysis of gear faults and the influence of gear fault size and load on the fault characteristics. 
Acknowledgement: This research was funded by Natural Science Foundation of Beijing, China (No. 3182005), National Natural Science Foundation of China (No. 51635001), and National Natural Science Foundation of China (No. 50235008).

\section{References}

Adam, G. (2016): Diagnostics of rotor damages of three-phase induction motors using acoustic signals and SMOFS-20-EXPANDED. Archives of Acoustics, vol. 41, no. 4, pp. 507-515.

Adam, G. (2018): Recognition of acoustic signals of commutator motors. Applied Science, vol. 8 , no. 12 .

Adam, G.; Witold, G. (2018): Vibration-based fault diagnosis of commutator motor. Shock and Vibration, vol. 2018.

Adam, G. (2019): Fault diagnosis of single-phase induction motor based on acoustic signals. Mechanical Systems and Signal Processing, vol. 117, pp. 65-80.

Almeida, L. B. (1994): The fractional Fourier transform and time-frequency presentations. IEEE Trans on Signal Processing, vol. 42, pp. 3084-3091.

Amaranth, M.; Praveen Krishna, I. R. (2012): Empirical mode decomposition of acoustic signals for diagnosis of faults in gears and rolling element bearings. Institution of Engineering and Technology, vol. 6, no. 4, pp. 279-287.

Bajric, R.; Zuber, N. S.; krimpas, G. A.; Mijatovic, N. (2016): Feature extraction using discrete wavelet transform for gear fault diagnosis of wind turbine gearbox. Shock and Vibration, vol. 2016.

Chen, H. F.; Sun, Y. Q.; Shi, Z. Y.; Lin, J. C. (2016): Intelligent analysis method of gear faults based on FRWT and SVM. Shock and Vibration, vol. 2016.

Chen, H. F.; Sun, Y. Q.; Shi, Z. Y.; Li, R.; Zhao, Y. et al. (2017): Gear fault feature extraction based on fractional fourier transform. Journal of the Chinese Society of Mechanical Engineers, vol. 38, pp. 639-645.

Chen, L.; Zhao, D. (2005): Optical image encryption based on fractional wavelet transform. Optics Communications, vol. 254, pp. 361-367.

Dadon, I.; Koren, N.; Klein, R.; Bortman, J. (2019): A step toward fault type and severity characterization in spur gears. Journal of Mechanical Design, Transactions of the ASME, vol. 141, no. 8, 083301.

Gargour, C.; Gabrea, M.; Ramachandran, V. (2009): A short introduction to wavelets and their applications. IEEE Circuits and Systems Magazine, vol. 9, no. 2, pp. 57-68.

Goch, G.; Ni, K.; Peng, Y.; Guenther, A. (2017a): Future gear metrology based on areal measurements and improved holistic evaluations. CIRP Annals, vol. 66, no. 1, pp. 469-474.

Huang, Y. (1988): The fractional wave packet transform. Multidimensional Systems and Signal Processing, vol. 4, pp. 399.

Huang, Z. W.; Liang, J.; Lei, L.; Hu, J. C. (2019): Detection of ring gear surface defects of wheel speed sensor based on neural network. Tenth International Symposium on Precision Engineering Measurements and Instrumentation, vol. 11053, 110533 U. 
Jiang, L. M.; Zhang, J. J.; Xuan, P.; Zou, Q. (2016): BP neural network could help improve pre-miRNA identification in various species biomed. BioMed Research International, vol. 2016.

Li, J. Y.; Han, C. F.; Yu, F. (2017): A new processing method combined with bp neural network for francis turbine synthetic characteristic curve. Research International Journal of Rotating Machinery, vol. 2017.

Mallat, S. G. (1989): A theory for multiresolution signal decomposition: the wavelet representation. IEEE Transactions on Pattern Analysis and Machine Intelligence, no. 11, pp. 674-693.

Mendlovic, D.; Zalevsky, Z.; Mas, D. (1997): Fractional wavelet transform. Applied Optics, vol. 36, no. 20, pp. 4801-4806.

Narayanan, V. A.; Prabhu, K. M. (2003): The fractional fourier transform: theory implementation and error analysis. Microprocessors and Microsystems, vol. 27, pp. 511-521.

Ni, K.; Peng, Y.; Stöbener, D.; Goch, G. (2019): Cylindrical gear metrology. Metrology, pp. 1-29.

Park, J.; Kim, Y.; Na, K. (2019): Variance of energy residual (VER): an efficient method for planetary gear fault detection under variable-speed conditions. Journal of sound and vibration, vol. 453, pp. 253-267.

Peng, Z. K.; Chu, F. L. (2004): Application of the wavelet transform in machine condition monitoring and fault diagnostics: a review with bibliography. Mechanical Systems and Signal Processing, vol. 18, pp. 199-221.

Qin, T. L.; Yang, Y.; Cheng, Q. (2008): Bearing fault diagnosis based on IMF energy moment and neural network. Vibration Testing and Diagnosis, vol. 28, pp. 229-232.

Sahoo, S.; Laskar, R. A.; Das, J. K.; Laskar, S. H. (2019): Gear fault diagnosis and classification using machine learning classifier. ACM International Conference Proceeding Series, pp. 69-72.

Samanta, B. (2004): Artificial neural networks and genetic algorithms for gear fault detection. Mechanical Systems and Signal Processing, vol. 18, no. 5, pp. 1273-1282.

Samanta, B.; Al-Balushi, K. R.; Al-Balushi, S, A. (2003): Artificial neural networks and support vector machines with genetic algorithm for bearing fault detection Engineering. Applications of Artificial Intelligence, vol. 16, pp. 657-665.

Shao, H. J.; Deng, X. (2018): AdaBoosting neural network for short-term wind speed forecasting based on seasonal characteristics analysis and lag space estimation. Computer Modeling in Engineering \& Sciences, vol. 114, no. 3, pp. 277-293.

Shi, Z. Y.; Lin, H.; Lin, J. C. (2013): Current status and trends of large gears metrology. Journal of Mechanical Engineering, vol. 49, pp. 35-44.

Tao, R.; Li, Y. L.; Wang, Y. (2010): Short-time fractional Fourier transform and its applications. IEEE Transactions on Signal Processing, vol. 58, pp. 2568-2580.

Wang, L. J.; Ji, S. F.; Ji, N. Y. (2019): Application of CSA-BP neural network in gearbox fault diagnosis. International Journal of Circuits, Systems and Signal Processing, vol. 13, pp. 273-280. 
Wiemann, A.; Stein, M.; Kniel, K. (2017): Traceability of gear measurements for large gear boxes. VDI Reports 2316, pp. 205-216.

Wood, J. C.; Barry, D. T. (1994): Linear signal synthesis using the Radon-Wigner transform. IEEE Transactions on Signal Processing, vol. 42, pp. 2105-2111.

Xu, B. Y.; Zhang, H. J.; Wang, Z. T.; Wang, H. X.; Zhang, Y. L. (2015): Model and algorithm of BP neural network based on expanded multi chain. Quantum Optimization Mathematical Problems in Engineering, vol. 2015.

Yang, Y.; Duan, J. B.; Yu, H. T.; Gao, Z. P.; Qiu, X. S. (2018): An image classification method based on deep neural network with energy model. Computer Modeling in Engineering \& Sciences, vol. 117, no. 3, pp. 555-575.

Yuan, L. (2008): Wavelet-fractional Fourier transform. Chinese Physics B, vol. 17, no. 1, pp. 170-176.

Zhao, J. G. (2018): Distance control algorithm for automobile automatic obstacle avoidance and cruise system. Computer Modeling in Engineering \& Sciences, vol. 116, no. 1, pp. 69-88.

Zhuo, Z. (2018): Novel image watermarking method based on FRWT and SVD. International Journal of Electronic Security and Digital Forensics, vol. 10, no. 1, pp. 97-107. 